\title{
Avances en el conocimiento y manejo de las dislipoproteinemias
}

\author{
FAUSTO GARMENDIA \\ Decano, Facultad de Medicina, UNMSM.
}

\begin{abstract}
RESUMEN
Se efectúa una revisión de los factores lipídicos y no lipìdicos de riesgo cardiovascular. Los factores lípídicos más importantes son el incremento del colesterol LDL, VLDL, IDL triglicéridos, lipoproteína(a) y colesterol no HDL, así como disminución del colesterol HDL, los cuales constituyen factores independientes de riesgo cardiovascular. Se clasifica las dislipoproteinemias en primarias y secundarias. Las dislipoproteinemias primarias son alteraciones innatas de carácter familiar o no familiar, dentro de las cuales destacan la hipercolesterolemia común, hiperlipidemia familiar combinada, hipercolesterolemia familiar, hipertrigliceridemia familiar, hiperlipidemia de remanentes de VLDL y la quilomicronemia primaria. Las dislipoproteinemias secundarias se producen como consecuencia de diversas enfermedades; entre las más comunes están el hipotiroidismo, diabetes mellitus, síndrome nefrótico, obstrucción biliar crónica, insuficiencia renal. Asimismo, existen algunos medicamentos o drogas que modifican el metabolismo lipídico, como el alcohol, beta-bloqueadores, diuréticos, progestágenos, corticosteroides, etc. Se enfatiza que la determinación del perfil lípido en ayunas no es suficiente para evaluar el metabolismo lipídico y se sugiere la conveniencia de realizar estudios posprandiales. El manejo terapéutico de estos trastornos debe considerar al régimen alimenticio, el ejercicio y la utilización de medicamentos, como los inhibidores de la hidroxi-metil-glutaril coenzima A y los derivados de los ácidos fíbricos. Se demuestra a través de megaestudios, multicéntricos, internacionales, que las intervenciones terapéuticas disminuyen la prevalencia de eventos cardiovasculares y la morbimortalidad respectiva.
\end{abstract}

Palabras clave: Lípidos; colesterol; lipoproteinas del colesterol HDL; lipoproteinas del colesterol LDL; lipoproteinas VLDL colesterol.

\section{ADVANCES IN THE KNOWLEDGE AND TREATMENT OF DYSLIPOPROTEINEMIAS SUMMARY}

A review of the lipidic and non-lipidic cardiovascular risk factors is done. The increase of LDL, VLDL, IDL cholesterol, non-HDL triglycerides and lipoprotein(a) as well as HDL cholesterol decrease were considered as main lipidic cardiovascular risk factors. Dyslipoproteinemias are classified as primary and secondary. Primary dyslipoproteinemias are inborn errors of lipid metabolism, familiar or non-familiar, including common hypercholesterolemia, combined familiar hyperlipidemia, familiar hypercholesterolemia, familiar hypertrigliceridemia, VLDL remanents hyperlipidemia and primary quilomicronemia. The secondary dyslipoproteinemias are produced by various diseases, including hypotiroidism, diabetes mellitus, nephrotic syndrome, chronic biliary obstruction, renal insufficiency and so on; there are some drugs that modify lipid metabolism such as alcohol, beta-blockers, diuretics, progestagens, corticosteroides, and many others. It is emphasized that the fasting lipid profile alone is not sufficient to determine the lipidic metabolic disorder, and it is suggested the convenience of conducting postprandial studies. In the therapeutic handling it is necessary to consider the diet and physical exercises; and when these measures are not sufficient, try to normalize the lipid profile by using drugs such as hydroxi-metil-glutaryl coenzime A inhibitors and fibric acids derivatives. Therapeutic interventions, supported by results of multicentric international mega-studies, have shown a decrease in the prevalence of the cardiovascular events and the related morbidity and mortality.

Key words: Lipids; cholesterol; lipoproteins, HDL cholesterol; lipoproteins, LDL cholesterol; lipoproteins, VLDL cholesterol.

Correspondencia:

Dr. Fausto Garmendia Lorena

Facultad de Medicina, UNMSM.

Av. Grau 755. Lima 1 - Perú

E-mail: garmendial@terra.com.pe 


\section{INTRODUCCIÓN}

Hemos preferido denominar dislipoproteinemias a los trastornos del metabolismo de los lípidos corporales, para significar que los lípidos que se mide en la sangre son en realidad fracciones lipídicas unidas a proteínas transportadoras (apoproteínas), que les permite disolverse en el medio acuoso de la sangre. De otra manera, circularían en forma de gotas de grasa, provocando embolias. La importancia de su estudio radica en el conocimiento que estas alteraciones incrementan el riesgo de acelerar el proceso de ateroesclerosis y, a través de ello, incrementar la prevalencia de eventos cardiovasculares (infarto del miocardio, accidentes cerebrovasculares, enfermedad vascular periférica o aneurismas aórticos ateroescleróticos), que llevan a la muerte.

Se debe puntualizar, sin embargo, que el riesgo cardiovascular no es incrementado sólo por la alteración de los lípidos, sino que es indispensable considerar también a factores no lipídicos (Tabla 1).

Los factores lipídicos de riesgo cardiovascular los podemos encontrar en la Tabla 2, en la que se puede apreciar que sólo una parte de ellos es mensurable por los métodos bioquímicos convencionales. Este hecho presta una importancia grande a la medición del colesterol No-HDL, que se calcula en una forma muy simple; ello se obtiene al restar al colesterol total la concentración del colesterol HDL. Esta fracción No-HDL engloba a todas las fracciones aterogénicas, incluidas aquellas no medibles habitualmente, como el colesterol IDL y la lipoproteína (a) (Tabla 2).

Antes de tratar sobre la clasificación de las dislipoproteinemias, se describe el perfil lipídico ideal para personas que tienen $0-1$ factores de riesgo cardiovascular $\left({ }^{1}\right)$ :

\footnotetext{
- Colesterol total (CT) : $<200 \mathrm{mg} / \mathrm{dL}$

- Colesterol LDL : $<130 \mathrm{mg} / \mathrm{dL}$

- Triglicéridos $(\mathrm{Tg}) \quad:<150 \mathrm{mg} / \mathrm{dL}$

- Colesterol HDL : $\quad>45 \mathrm{mg} / \mathrm{dL}$

- Colesterol No-HDL : $<160 \mathrm{mg} / \mathrm{dL}$
}

Tabla 1.- Factores no lipídicos de riesgo cardiovascular.

- Hombres > 45 años.

- Mujeres posmenopáusicas.

- Sedentarismo.

- Historia familiar de enfermedad cardiovascular.

- Obesidad abdominal.

- Diabetes mellitus.

- Hipertensión arterial.

- Tabaquismo.

- Homocisteína elevada.

- Infección por chlamydia.

- Resistencia a la insulina.

- Signos clínicos de enfermedad vascular periférica (EVP).

- Elevación de proteína $\mathrm{C}$ reactiva.

- Incremento de leucocitos y hematócrito.

- Incremento de fibrinógeno, factor VIII, factor VII.

- Incremento del inhibidor del activador del plasminógeno I.

- Resistencia a la inactivación de la proteína C de los factores V y VIII.

- Genotipo DD para la enzima convertidora de angiotensina (ECA).

- Signos clínicos de hiperlipidemia.

- Deficiencia de vitaminas antioxidantes.

- Síndrome plurimetabólico.

Tabla 2.- Factores lipídicos de riesgo cardiovascular.

- Incremento de colesterol LDL, VLDL, IDL.

- Disminución de HDL.

- Hipertrigliceridemia.

- Incremento del colesterol No-HDL.

- Incremento de lipoproteína (a).

- Incremento de LDL pequeñas y densas.

Para calcular las fracciones LDL y VLDL se utiliza la fórmula de Fridewald, siempre que la concentración de Tg no exceda $400 \mathrm{mg} / \mathrm{dL}$, que consiste en:

$\mathrm{VLDL}=\mathrm{Tg} / 5 ; \quad \mathrm{LDL}=\mathrm{CT}-\mathrm{HDL}-\mathrm{VLDL}$ 
Tabla 3.- Dislipoproteinemias primarias. Clasificación y características de las hiperlipidemias primarias (géneticas) $\left(^{2}\right)$.

\begin{tabular}{|c|c|c|c|c|c|c|}
\hline \multirow[b]{2}{*}{$\begin{array}{l}\text { Enfermedad } \\
\text { incrementada }\end{array}$} & \multirow[b]{2}{*}{ Defecto molecular } & \multicolumn{3}{|c|}{ Efecto en los niveles lipídicos } & \multirow[b]{2}{*}{$\mathrm{ECC}$} & \multirow[b]{2}{*}{ Pancreatitis } \\
\hline & & Lipoproteina (s) & Colesterol & Triglicéridos & & \\
\hline $\begin{array}{l}\text { Hipercoleste- } \\
\text { rolemia común }\end{array}$ & $\downarrow$ Receptores LDL & LDL & $\uparrow$ & $\leftarrow$ & + & - \\
\hline $\begin{array}{l}\text { Hiperlipidemia } \\
\text { familiar com- } \\
\text { binada }\end{array}$ & $\begin{array}{l}\uparrow \text { Secreción hepática } \\
\text { de VLDL } \\
\text { Apo- B }\end{array}$ & $\begin{array}{l}\text { VLDL } \\
\text { LDL }\end{array}$ & $\uparrow$ & $\uparrow$ & ++ & - \\
\hline $\begin{array}{l}\text { Hipercoleste- } \\
\text { rolemia familiar }\end{array}$ & $\downarrow$ Receptores LDL & LDL & $\uparrow \uparrow$ & $\leftarrow$ & +++ & - \\
\hline $\begin{array}{l}\text { Hipertriglice- } \\
\text { ridemia familiar }\end{array}$ & $\begin{array}{l}\uparrow \text { Secreción hepática } \\
\text { de VLDL } \\
\downarrow \text { Catabolismo VLDL }\end{array}$ & VLDL & $\uparrow \uparrow$ & $\uparrow \uparrow$ & - & +- \\
\hline $\begin{array}{l}\text { Hiperlipidemia } \\
\text { remanente }\end{array}$ & $\downarrow$ Catabolismo IDL & IDL & $\uparrow \uparrow$ & $\uparrow \uparrow$ & +++ & +- \\
\hline $\begin{array}{l}\text { Quilomicro- } \\
\text { nemia primaria }\end{array}$ & $\begin{array}{l}\downarrow \text { Catabolismo del } \\
\text { quilomicrón }\end{array}$ & $\begin{array}{l}\text { Quilomicrones } \\
\text { VLDL }\end{array}$ & $\uparrow$ & $\uparrow \uparrow \uparrow$ & - & +++ \\
\hline
\end{tabular}

ECC: Enfermedad cardíaca coronaria.

La clasificación fisiopatológica de las dislipoproteinemias comprende a las primarias y secundarias. Las dislipoproteinemias primarias son trastornos innatos del metabolismo lipídico; pueden ser familiares o no familiares (Tabla 3).

Las dislipoproteinemias secundarias se producen como consecuencia de un desorden subyacente que altera el metabolismo intermediario (Tabla 4).

Cuando hemos examinado pacientes con enfermedad cardiaca coronaria (ECC) definida, en el Hospital Nacional Dos de Mayo $\left(^{3}\right)$, la mayoría tuvo disminución del HDL e incremento del colesterol No HDL, y no como se suele señalar incremento del colesterol LDL. Esto comprueba que se debe considerar a factores lipídicos diferentes a los clásicos para evaluar el grado de riesgo cardiovascular, cuyo sustento científico analizaremos a continuación.

\section{INCREMENTO DEL COLESTEROL LDL}

En el estudio de Framingham, de $1986\left({ }^{4}\right)$, se pudo demostrar que el incremento del colesterol total, cuyo mayor representante es el colesterol LDL, por encima de $200 \mathrm{mg} / \mathrm{dL}$ incrementa progresiva y significativamente la incidencia de ECC por cada 1000 personas. Del mismo modo, el Multiple Risk Factors Intervention Trial (MRFIT), realizado en 356,222 personas mostró en forma clara que el

Tabla 4.- Dislipoproteinemias secundarias.

\begin{tabular}{ll} 
- Hipotiroidismo & - Lipodistrofia \\
- Diabetes mellitus & - Obesidad \\
- Síndrome nefrótico & - Drogas y medicamentos \\
- Obstrucción biliar crónica & - Alcohol \\
- Insuficiencia renal & - Beta-bloqueadores \\
- Enfermedad de von Gierke & - Diuréticos \\
- Mielomatosis & - Progestágenios \\
- Porfiria aguda & - Corticosteroides \\
\hline
\end{tabular}


incremento del CT de 200 a $250 \mathrm{mg} / \mathrm{dL}$ incrementa el riego relativo de 1 a 2 y que con $300 \mathrm{mg} / \mathrm{dL}$ el riesgo se cuatriplica $\left({ }^{5}\right)$.

\section{DISMINUCIÓN DEL COLESTEROL HDL}

Las personas que tienen concentraciones bajas de HDL, menores de $45 \mathrm{mg} / \mathrm{dL}$, tienen mayor riesgo de ECC que aquellas con concentraciones superiores. Inclusive, se considera que concentraciones superiores a $60 \mathrm{mg} / \mathrm{dL}$ tienen riesgo negativo $\left({ }^{6}\right)$. Esta acción protectora está vinculada al papel fisiológico de esta fracción lipoproteica, que transporta el colesterol LDL de los tejidos, llámese vasos arteriales, hacia el hígado y su subsecuente degradación. Entre otras acciones beneficiosas, en este sentido se describe las siguientes:

1. Disminuir el efecto vasoconstrictor de la acetilcolina $\left.{ }^{7}\right)$.

2. Disminuir la formación de metaloproteinasas, enzimas que favorecen la inestabilidad de la placa ateromatosa $\left({ }^{8}\right)$.

3. Inhibición de la oxidación de las LDL, con lo que las hace menos pasibles de ser fagocitadas por los macrófagos, y la consiguiente formación de las células espumosas, base estructural de las placas ateromatosas de los vasos arteriales $\left({ }^{9}\right)$.

\section{CONCENTRACIÓN ELEVADA DE TRIGLICÉRIDOS}

La hipertrigliceridemia es un factor independiente de riesgo cardiovascular, que se incrementa aún más cuando el paciente tiene además concentraciones bajas de HDL, situación muy frecuente. Haim y col. $\left({ }^{10}\right)$ han demostrado que, a tanto mayor la concentración de triglicéridos, por encima de $150 \mathrm{mg} / \mathrm{dL}$, tanto mayor es el riesgo de morir por eventos cardiovasculares, tanto en mujeres como en varones. Otros autores han demostrado esta misma evidencia en varios trabajos $\left({ }^{11-16}\right)$.

\section{¿ES SUFICIENTE MEDIR EL PERFIL LIPÍDICO EN AYUNAS?}

De acuerdo a nuestras investigaciones no es suficiente, pese a que es común evaluar el perfil lipídico en ayunas. Así, cuando hemos efectuado una evaluación metabólica durante 6 horas después de la administración de un desayuno, con $730 \mathrm{kcal}$, de las cuales $45 \%$ corresponde a grasas, $37 \%$ a hidratos de carbono y $7 \%$ a proteínas, se ha encontrado información adicional muy valiosa. En un grupo de obesos, en comparación con normales, se ha podido establecer que en la muestra basal se encontró cifras significativamente mayores de $\mathrm{Tg}$ y menores de HDL; en el período posprandial se demostró hiperinsulinemia, una mayor elevación de $\mathrm{Tg}$, disminución mayor de HDL y cambios en la movilización de los ácidos grasos no esterificados; que, en conjunto, permiten afirmar que el obeso, después de la ingestión de alimentos, se encuentra en una condición metabólica de mayor riesgo cardiovascular que cuando se considera sólo los valores basales $\left(^{3,17}\right)$.

\section{MANEJO DE LAS DISLIPOPROTEINEMIAS}

Los pilares fundamentales del tratamiento de las dislipoproteinemias son el régimen alimenticio y la actividad física. El régimen alimenticio está orientado a proporcionar el número adecuado de calorías para mantener un peso normal. Esto, en pacientes con obesidad, es sumamente importante, dado que la obesidad determina el incremento de triglicéridos y disminución del colesterol HDL. Se debe considerar 1 gramo de proteínas por $\mathrm{kg}$ de peso normal o ideal; $30 \%$ del valor calórico total en forma de grasas, de las cuales una proporción importante debe estar a base de aquellas que tienen un alto contenido de ácidos grasos poliinsaturados (aceite de maíz, soya, oliva). Se debe recomendar la limitación de la ingesta de alimentos ricos en colesterol (vísceras, grasas de origen animal, yema de huevo, embutidos, mantequilla, grasa de la leche, etc), para procurar una ingesta diaria de colesterol 
menor de $300 \mathrm{mg}$; así mismo, se debe insistir en la ingesta de fibra, presente en vegetales, legumbres, frutas, granos enteros, de la cual la alimentación peruana es rica.

Se ha demostrado que la actividad física determina una disminución de los triglicéridos $\left({ }^{18,19}\right)$, incrementa el colesterol HDL y tiene un menor efecto sobre la disminución de colesterol LDL.

Cuando estas medidas no son suficientes para normalizar el perfil lipídico, es indispensable utilizar medicamentos hipolipemiantes (Tabla 5).

Con fines prácticos, debe considerarse a los derivados de los ácidos fíbricos para el tratamiento de los pacientes con hipertrigliceridemia. El gemfibrozil, a una dosis de 600 a $1200 \mathrm{mg}$ diarios, ha demostrado un efecto muy beneficioso $\left({ }^{20}\right)$.

En los pacientes con hipercolesterolemia, los medicamentos de mayor uso son las estatinas, de las cuales la simvastatina es la que más se ha estudiado y ha demostrado muy buen efecto, usada a una dosis que varía entre 20 y $80 \mathrm{mg}$ diarios.

Cuando la monoterapia con los medicamentos anteriores no es suficiente, se puede combinar con atrapadores intestinales de colesterol, como colestipol o colesteramina, a las dosis de 6 a $12 \mathrm{~g}$ diarios. Recientemente se ha introducido en el mercado nacional la ezetimiba a la dosis de $10 \mathrm{mg}\left({ }^{21}\right)$, que también disminuye la absorción intestinal de colesterol. El ácido nicotínico y el probucol no tienen mayor uso por sus efectos secundarios, particularmente en nuestro medio.

\section{RESULTADO DE LAS INTERVENCIONES}

Los estudios de Helsinki ( $\left.{ }^{20}\right)$, el Scandinavian Simvastatin Survival Study (4S) $\left({ }^{22}\right)$, Goalls $\left({ }^{23}\right)$ son ejemplos que demuestran la eficacia y seguridad en la reducción de los lípidos sanguíneos; pero, particularmente el efecto de disminuir la prevalencia de eventos cardiovasculares y de disminuir la morbimortalidad por dichos
Tabla 5.- Drogas hipolipemiantes.

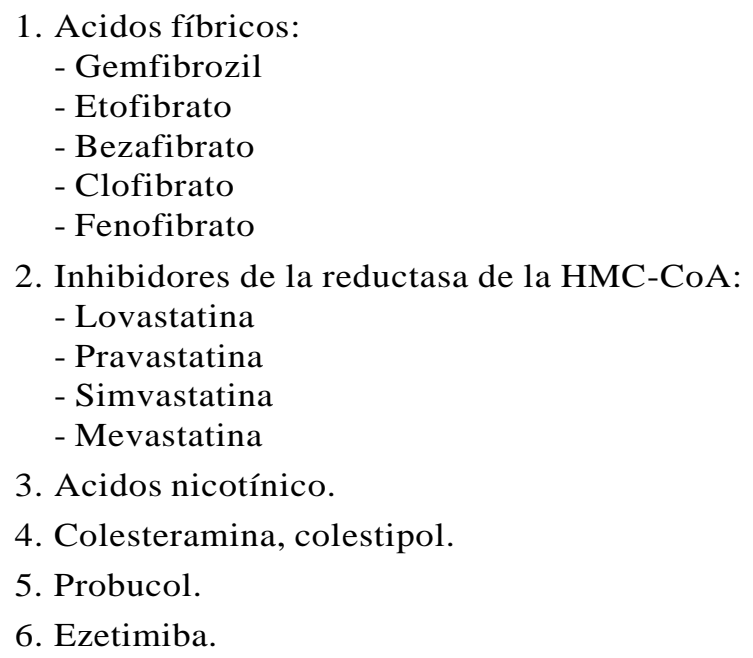

2. Inhibidores de la reductasa de la HMC-CoA:

- Lovastatina

- Pravastatina

- Simvastatina

- Mevastatina

3. Acidos nicotínico.

4. Colesteramina, colestipol.

5. Probucol.

6. Ezetimiba.

eventos, tanto en intervenciones primarias como secundarias. En el caso de las estatinas, se ha demostrado que su efecto en la reducción de eventos cardiovasculares no sólo se sustenta en su efecto hipolipemiante, sino que ejerce otros efectos pleotrópicos. Se ha demostrado que mejoran la función endotelial, tienen efecto antiinflamatorio, estabilizan la placa ateromatosa, disminuyen el PAI -1 , incrementan la densidad ósea y tienen efecto favorable sobre la enfermedad de Alzheimer.

\section{CONCLUSIONES}

- Existen factores lipídicos y no lipídicos de riesgo cardiovascular.

- El incremento de LDL no es el único factor de riesgo cardiovascular.

- La disminución de cHDL es un factor de riesgo muy importante.

- La hipertrigliceridemia es un factor de riesgo independiente, se acompaña de disminución del cHDL.

- Los derivados de los ácidos fíbricos tienen un efecto marcado para disminuir los triglicéridos. 
- Las estatinas disminuyen el cLDL, ApoB, lipoproteína (a), triglicéridos e incrementan el cHDL.

- A tanto más altos los triglicéridos y más bajo el cHDL, el efecto de las estatinas es mayor.

- Las estatinas tienen varios efectos pleotróficos.

- Las estatinas difieren entre sí en sus efectos terapéuticos $\left({ }^{24}\right)$.

- El régimen alimenticio, actividad física y cambio del estilo de vida son los pilares fundamentales en la prevención de la patología cardiovascular.

Estudio realizado con financiamiento del Fondo de Desarrollo Universitario (FEDU), UNMSM, 2000 y de Merck Sharp \& Dohme.

\section{BIBLIOGRAFÍA}

1. Expert Panel on Detection, Evaluation and Treatment of High Blood Colesterol in Adults. Executive Summary of the Third Report of the National Cholesterol Education Program (NCEP), Expert Panel on Detection, Evaluation, and Treatment of high blood cholesterol in adults (Adult Treatment Panel III). JAMA 2001; 285: 2486-97.

2. Davignon J, Genest J Jr. Genetics of lipoprotein disorders. Endocrinol Metab Clin North America 1998; 27: 521-50.

3. Pando R, Garmendia F, Armas J, Torres W. Estudio de factores de riesgo en pacientes con enfermedad coronaria. XI Congreso Nacional y XXII Curso Internacional de Medicina Interna. Libro de Resúmenes. Lima, nov 2000: 111.

4. Castelli WP, Garrison RJ, Wilson PW, Abbott RD, Kalousdian S, Kannel WB. Incidence of coronary heart disease and lipoprotein cholesterol levels. The Framingham Study. JAMA 1986; 256: 2835-8.

5. Stamler J, Wentworth D, Neaton J, the MRFIT RESEARCH group. Is relationship between serum cholesterol and risk/of premature death from coronary heart disease continuous and graded? JAMA 1986; 256: 2823-8.

6. Kitamura A, Iso H, Naito Y, Iida M, Konishi M, Folsom $\mathbf{A R}$, et al. High-density lipoprotein cholesterol and premature coronary heart disease in urban Japanese men. Circulation 1994; 89: 2533-9.

7. Zeiher AM, Schachlinger V, Hohnloser SH, Saurbier B, Just H. Coronary atherosclerotic wall thickening and vascular reactivity in humans. Elevated high-density lipoprotein levels ameliorate abnormal vasoconstriction in early atherosclerosis. Circulation 1994; 89: 2525-32.

8. Xu XP, Meisel SR, Ong JM, Kaul S, Cercek B, Rajavashisth TB, et al. Oxidized low-density lipoprotein regulates matrix metalloproteinase- 9 and its tissue inhibitor in human monocyte-derived macrophages. Circulation 1999; 99: 993-8.

9. Parthasarathy S, Barnett J, Fong LG. High-density lipoprotein inhibits the oxidative modification of low-density lipoprotein. Biochim Biophys Acta 1990; 1044: 275-83.

10. Haim M, Benderly M, Brunner D, Behar S, Graff E, Reicher-Reiss H, et al. Elevated serum triglyceride levels and long-term mortality in patients with coronary heart disease. Circulation 1999; 100: 475-82.

11. Ginsberg HN. Hypertriglyceridemia: New insights and new approaches to pharmacology therapy. Am J Cardiol 2001; 87: $1174-80$.

12. Gaziano JM, Hennekens CH, O'Donnell CJ, Breslow JL, Buring JE. Fasting triglycerides, high-density lipoprotein, and risk of myocardial infarction. Circulation 1997; 96: 2520-5.

13. Grundy SM. Hypertriglyceridemia, atherogenic dyslipidemia, and the metabolic syndrome. Am J Cardiol 1998; 81: 18B-25B.

14. Krauss RM. Atherogenicity of triglyceride-rich lipoproteins. Am J Cardiol 1998; 81: 13B-17B.

15. Cullen $\mathbf{P}$. Evidence that triglycerides are an independent coronary heart disease risk factor. Am J Cardiol 2000; 86:943-9.

16. Krauss RM. Triglycerides and atherogenic lipoproteins: rationale for lipid management. Am J Med 1998; 105: 58S$62 \mathrm{~S}$.

17. Garmendia F, Crispin S, Flores $\mathbf{R}$ y col. Lipemia postprandial en obesos. II Jornadas Científicas de Investigación en Salud. Facultad de Medicina, UNMSM, Libro de Resúmenes, Lima, 2000: 132.

18. Hardaman A, Lawrence JEM, Herd SL. Postprandial lipemia in endurance trained people during a short interruption to training. J Appl Physiol 1998; 84(6): 1895-901.

19. Weintraub MS, Rosen Y, Otto R, Eisenberg S, Breslow JL. Physical exercise conditioning in absence of weight loss reduce fasting and postprandial triglyceride-rich lipoprotein levels. Circulation 1989; 79: 1007-14.

20. Frick MH, Elo O, Haapa K, Heinonen OP, Heinsalmi P, Helo P, et al. Helsinki Heart Study: primary-prevention with Genfibrozil in middle-aged men with dyslipidemia. N Engl J Med 1987; 317: 1237-45.

21. Sudhop T, Lutjohann D, Kodal A, Igel M, Tribble DL, Shah S, et al. Inhibition of intestinal cholesterol absorption by Ezetinibe in humans. Circulation 2002; 106: 1943.

22. The Scandinavian Simvastatin Survival Study Group (4S). Randomized trial of cholesterol lowering in 4444 patients with coronary heart disease. The Lancet 1994; 344 (8934): 1383-9.

23. Garmendia F, Brown AS, Reiber I, Adams PC. Attaining Unites States and European guideline LDL-cholesterol levels with simvastatin in patients with coronary heart disease (The GOALLS study). Curr Med Res Opin 2000; 16(3): 208-19.

24. Furberg CD. Natural statins and stroke risk. Circulation 1999; 99: 185-8. 